ISSN: 2007-7033 | Núm. 54 | e1011 | Sección abierta: artículo teórico (revisión sistemática) |

\title{
Implicación familiar en el bachillerato: una estrategia para favorecer la permanencia escolar
}

\section{Family involvement in high school: A strategy to improve student permanency}

\author{
ALEJANDRA INFANTE BLANCO* \\ LAURA ElENA PADILla GonZÁLEZ*
}

Es amplia la literatura que aborda la relación familia-escuela durante la educación básica, pero es menor la que estudia la temática desde la educación media superior o superior. Este trabajo presenta el desarrollo del concepto de implicación familiar en la educación y su importancia en el logro académico de los estudiantes de bachillerato; se asume que la familia tiene un rol fundamental en el acompañamiento socioafectivo y cognitivo que puede brindar al adolescente durante esta etapa, lo que facilita su permanencia escolar. Con base en el modelo de Epstein, se clasifican los niveles de implicación familiar, así como las prácticas familiares y escolares que dan lugar al logro académico de los estudiantes; se analizan algunas estrategias identificadas en la literatura que pueden ser de utilidad para fomentar la implicación de las familias en las preparatorias mexicanas a fin de coadyuvar a uno de sus mayores retos: la permanencia de los estudiantes en el bachillerato.

A growing body of research confirms the importance of the family-school relationship during the early and elementary childhood education; however, scant research has addressed this topic with regard to high school education. This paper focuses on the concept of family involvement in education and explores its relationship to high school students' outcomes; it assumes that family involvement in education remains central in this stage, adolescence, as students continue to grow up, as well as for its strategic role in promoting school retention in this level of education. This review relies on the Epstein's model, which suggests different levels of family involvement; it also points out family and school practices that promote students' outcomes. Based on this, we analyze some strategies identified in the literature that may be helpful for Mexican high schools in order to improve family involvement, which in turn could contribute to face one of the challenges of this educational level: high school students' retention.

\section{Palabras clave:}

implicación familiar, educación media superior, logro educativo, permanencia escolar

\section{Keywords:}

family involvement, high school education, student outcomes, student permanency

Recibido: 17 de junio de 2019 | Aceptado para su publicación: 29 de enero de 2020

Fecha de publicación: 11 de febrero de 2020

Recuperado de: https://sinectica.iteso.mx/index.php/SINECTICA/article/view/1011

doi: 10.31391/S2007-7033(2020)0054-006

\footnotetext{
* Doctoranda en Investigación Educativa por la Universidad Autónoma de Aguascalientes. Correo electrónico: aleinfanteb@gmail.com

** Doctora en Educación por la Claremont Graduate University. Profesora-investigadora del Departamento de Educación del Centro de Ciencias Sociales y Humanidades de la Universidad Autónoma de Aguascalientes. Miembro del Sistema Nacional de Investigadores. Sus líneas de investigación son la política educativa y la gestión en educación media superior y superior. Correo electrónico: lepadill@gmail.com
} 


\section{INTRODUCCIÓN}

T a educación media superior (EMS) en México constituye un espacio formativo relevante para la vida de los jóvenes y el desarrollo del país. Entre sus finalidades se encuentran promover capacidades sociales que resulten en la incorporación de ciudadanos responsables, capaces de contribuir a la consolidación de la democracia; producir oportunidades de desarrollo económico; y favorecer el desarrollo de capacidades cognitivas, actitudinales y prácticas con las cuales sus egresados puedan acceder a empleos calificados. Con el cumplimiento de estos objetivos, se espera disminuir la pobreza, alcanzar equidad y expandir las posibilidades de progreso científico, tecnológico, humanístico y artístico que el país requiere para enfrentar los retos del siglo XXI (Bustamante, 2014).

Desde esta perspectiva, la educación de los bachilleres es una tarea compleja a la que se suman otras situaciones que caracterizan a la EMS: por un lado, la política que decreta la obligatoriedad (2012), con la que se espera que todos los jóvenes en edad de cursar la preparatoria tengan acceso al servicio educativo; por otro, el índice de deserción más alto de todo el sistema educativo: 14.4\% (INEE, 2017). Sirva esta contextualización para adentrarse en la idea de concebir a las familias como aliadas en la consecución de los objetivos de la EMS.

Brindar educación media superior de calidad para todos los jóvenes no puede ser una tarea que la escuela realice de manera aislada; que los estudiantes no solo ingresen, sino que permanezcan, alcancen una mejora en los aprendizajes y egresen, es un asunto que también compete a las familias, pues, como señalan Reparáz y Naval (2014), las familias son las receptoras últimas de la educación; por lo tanto, su participación es vital en el logro de la calidad educativa. Destacar su papel como actor educativo e involucrarlo en la dinámica escolar puede ser una opción insustituible para proveer a los jóvenes de una educación relevante (Martínez, 2012).

Aunque a través de los niveles educativos la presencia de la familia parece desdibujarse, esta sigue siendo importante tanto para el éxito escolar como para el desarrollo de los estudiantes; en realidad, su participación adquiere distintos matices que se representan en prácticas diversas dependiendo del nivel escolar, del tipo de financiamiento de la escuela e, incluso, de las costumbres de la comunidad. Estas ideas han sido apoyadas por diversos estudios, los cuales indican la relevancia de mantener cercana colaboración durante toda la escolarización (Comellas et al. 2013; Hernández, Gomariz, Parra y García, 2016; O’Shea, 2016).

El estudio de la implicación familiar en educación media superior permite conocer cómo esta se vincula a variables como el capital social (provisto por las familias), los niveles de logro educativo alcanzados por los estudiantes y los índices de deserción escolar en este tipo educativo.

Los principales hallazgos en torno a la temática, como explicamos más adelante, identifican la influencia de las expectativas de los padres hacia la escuela y la propia educación de sus hijos, el ambiente de aprendizaje en el hogar, la relación padres e hijo o hija, así como la disciplina y la implicación parental en actividades escolares y relativas al aprendizaje en el hogar; todo ello como factores asociados al logro educativo y, de este modo, a la permanencia en la escuela (Castro et al., 2015; Fan \& Chen, 2001; Fernández-Alonso, Álvarez-Díaz, Woitschach, Suárez-Álvarez \& Cuesta,

Infante / Padilla. Implicación familiar en el bachillerato: una estrategia para favorecer la permanencia escolar Sinéctica 54 www.sinectica.iteso. $m x$ 
2017; Flecha, 2015; Huerta, 2009; Jeynes, 2007, 2011; Loya, Arzola y Armendáriz, 2013; Pomerantz, Moorman \& Litwack, 2007; Redding, 2006; Sanders \& Epstein, 2005; Wilder, 2014). Sin embargo, aún hace falta investigar cuáles son las condiciones de participación familiar promovidas desde los bachilleratos y las prácticas con las que las familias se comprometen. De ahí que subrayemos la importancia de explorar el estado de la implicación familiar en la educación media superior.

Este trabajo tiene como objetivo mostrar una revisión teórica sobre el concepto de implicación familiar (family involvement) en relación con el aprendizaje de los estudiantes de bachillerato y como estrategia que promueve la permanencia escolar. Para ello, realizamos una búsqueda de información en bases de datos (EBSO, ERIC, Scielo, Redalyc y Google Académico) y consultamos las páginas del Instituto Nacional para la Evaluación de la Educación y de la Subsecretaría de Educación Media Superior; la fecha de búsqueda se acotó de 2001 a 2018; los descriptores utilizados fueron: parent involvement in high school, family involvement in middle school, relación familia escuela y participación familiar en la educación media superior, y sus combinaciones. Con estos, los buscadores arrojaron también resultados con los términos parent participation y parent engagement.

Durante la selección de documentos, favorecimos aquellos de contenido académico que contaran con referentes teóricos, metodológicos, resultados y conclusiones, presentados en forma de artículos de revistas, libros, informes, memorias de congreso, tesis, en especial los que tuvieran relación con la temática en nivel medio superior.

Considerando los criterios mencionados, en este trabajo recuperamos el análisis de 47 documentos, los cuales fueron revisados a partir de su relación con el modelo de Epstein. Los resultados se organizan de acuerdo con los cuatro apartados de este artículo: las aportaciones al concepto de implicación familiar en educación media; la implicación familiar como mecanismo para favorecer la permanencia escolar; la implicación familiar y el logro académico en el bachillerato; y los programas de intervención exitosos que promueven la implicación familiar.

Con base en el análisis de la información, en adelante usamos los términos implicación e involucramiento de forma indistinta. De igual modo, al estar centrado en el bachillerato, proponemos extender el término implicación parental, que destaca la participación de padres y madres, hacia una concepción en que esta se entiende como un subconjunto de la implicación familiar, es decir, se considera que otros miembros de la familia, además de los padres, puedan intervenir en el desarrollo y aprendizaje de los bachilleres.

\section{EL CONCEPTO DE IMPLICACIÓN FAMILIAR EN EDUCACIÓN MEDIA SUPERIOR}

Los resultados académicos de los estudiantes son producto de varios procesos e instituciones; esto significa que las características individuales de los estudiantes, las interacciones familiares, escolares y la relación entre ambas se entrelazan para, finalmente, alcanzar los aprendizajes que se pueden ver cristalizados en el logro académico; es decir, el involucramiento familiar solo es un factor asociado a los aprendizajes, por lo cual sería reduccionista pensar que estos se deben de manera exclusiva al apoyo de la familia, sin considerar los demás elementos y actores. 
No obstante, existe un cuerpo significativo de investigaciones cuyos hallazgos científicos han establecido una asociación entre implicación familiar y resultados académicos. Más aún, podríamos considerar que esta no solo tiene efectos positivos en el logro de aprendizajes, ya que la influencia positiva que esta conlleva se puede apreciar en la mejora de otros aspectos de los centros educativos y del desarrollo de los estudiantes y las comunidades.

En lo anterior coinciden Desforges y Abouchaar (2003), quienes explican que el concepto de implicación o involucramiento familiar (parent involvement) es multidimensional, esto es, incluye una serie de acciones diferenciadas, pero que pueden entenderse como un mismo concepto, por ejemplo, la supervisión en casa, apoyo con las tareas escolares, cercanía con los profesores, asistencia a las actividades escolares o tomar parte en las decisiones y gestiones escolares. Esta multidimensionalidad genera inconsistencias en la delimitación conceptual, pues las acciones pueden estar encaminadas a mejorar los aprendizajes de los educandos, o bien, a la gestión de los recursos de la escuela, de ahí que se vuelva necesario mirar hacia una conceptualización que distinga finalidades.

De acuerdo con Simon (2004), en el bachillerato, el involucramiento familiar disminuye por dos motivos: uno, los padres pueden asumir que, a medida que sus hijos crecen, necesitan más autonomía y menos orientación de los adultos y, por lo tanto, deliberadamente reducen su participación; y dos, los padres creen que no dominan los contenidos, por lo que dejan de intervenir en la realización de tareas escolares. Además, en este nivel educativo, prevalece la idea de que cuanto más se involucre a las familias, es más probable que estas brinden apoyo para el aprendizaje de los estudiantes, pero, aunque los padres quieren que sus hijos tengan éxito en la escuela, no todos tienen claro qué deben hacer para apoyarlos. En consecuencia y como parte de la delimitación del concepto, se percibe una distinción entre la participación de las familias en actividades organizadas por la escuela (relación familia-escuela) y la implicación en acciones que posibiliten aprendizajes y desarrollo de los bachilleres.

Una cantidad importante de la literatura centra su atención en la implicación de padres y madres en el logro académico, de ahí el concepto de parent involvement. Los estudios se han desarrollado, principalmente, en educación básica, en la cual los progenitores tienen mayor protagonismo. No obstante, en el caso del nivel medio superior, además de padres y madres, otros miembros, como hermanos, abuelo, tíos, primos, pareja, entre otros, participan en los procesos escolares de los estudiantes. Atendiendo a esta razón y a la consideración de una amplia diversidad en la conformación de las familias actuales, en este trabajo proponemos hablar de involucramiento familiar.

La investigación sobre la implicación familiar y el logro académico surge desde el interés de las disciplinas sociales por analizar la estructura y el funcionamiento familiar en relación con las calificaciones obtenidas por los estudiantes en la escuela. Pronto la participación de los padres se convirtió en un campo importante también para la investigación educativa; los estudios más destacados asociaban la presencia de la familia como un factor del éxito de las escuelas. El Informe Coleman (1966) es una prueba de ello, pues, con su carácter de líder en los estudios educativos de esa época, transformó la manera de ver a la familia y demostró que es un agente activo para el óptimo funcionamiento de la escuela (Jeynes, 2011). 
A partir de estos estudios, se sugiere que las familias y la escuela colaboren de manera activa, no solo para alcanzar los estándares deseados de aprendizaje, sino para promover ambientes que posibiliten el desarrollo de las capacidades cognitivas, emocionales y sociales.

En este sentido, la perspectiva de Epstein (2010) propone una mirada sistémica que comprende a la familia, la escuela y la comunidad como contextos relevantes para el desarrollo de los aprendizajes; postula que cuantas más interacciones existan entre estos escenarios, habrá más comunidades educativas que destaquen la importancia de la educación en la vida de los estudiantes y, con ello, se obtendrán resultados favorables no solo para las escuelas, sino para las familias y la comunidad misma.

La mirada sistémica de los contextos educativos subraya que las interacciones entre familia y escuela son bidireccionales (Egido, 2015; Glasgow \& Whitney, 2009); es decir, la disposición para entablar una relación cordial es de ambas (familia y escuela), pues sus efectos pueden llegar a rebasar la intención de favorecer ambientes de aprendizaje para los estudiantes. Esta idea se apoya en dos argumentos: en el primero hay una deseabilidad de que tanto la familia como la escuela muestren iniciativa para establecer un vínculo; se suma la postura de Simon (2004), quien afirma que la implicación familiar en la educación escolarizada es un asunto de dos voluntades, que corresponde tanto a las familias como a los bachilleratos generar condiciones que posibiliten el desarrollo de las capacidades cognitivas, sociales y afectivas de los niños y jóvenes.

El segundo argumento confirma la naturaleza permeable de la participación de las familias en la educación (Fernández-Alonso et al., 2017), al demostrar que esta no solo tiene efectos positivos en los estudiantes, sino que estos se extienden hacia las propias familias y los centros escolares.

El concepto de implicación familiar ha sido abordado por Jeynes (2007), cuya propuesta es referente conceptual en los estudios más recientes, porque recupera distintos aspectos para formular su definición; entre ellos se encuentran las expectativas sobre logros académicos, asistencia a actividades escolares, comunicación intrafamiliar, y supervisión de conducta y tareas como prácticas. Así, la implicación familiar se resume como "participación de los procesos y experiencias educativos" (p. 83). En esta definición se observa que la implicación familiar prevé una serie de actividades que la delimitan con claridad en el desarrollo del educando; además de aspectos escolares, los procesos y las experiencias educativos incluyen la relación al interior de las familias, lo que, finalmente, podría ser un reflejo del vínculo que se establece con la escuela.

La revisión de la literatura permite advertir que los autores se adhieren a dos acepciones del concepto involucramiento familiar: la primera pondera los efectos positivos sobre logro académico de los estudiantes (Hill \& Tyson, 2009; Jeynes, 2011; Wilder, 2014) y la segunda recupera aspectos ligados al absentismo, transición entre niveles educativos y aspectos del desarrollo social, afectivo y cognitivo (Castro et al., 2015; Mac Iver, Epstein, Sheldon \& Fonseca, 2015).

La aportación de Hill y Tyson (2009) refiere la implicación familiar como "interacciones de los padres con escuelas y con sus hijos para promover el éxito académico" 
(p. 741); aunque no detallan qué actividades caben en esa interacción, deja claro el motivo. Esta pudiera considerarse una definición acotada, ya que el propósito se explica únicamente como el éxito académico.

Una perspectiva complementaria es la definición de Castro et al. (2015) en la cual se considera la implicación familiar como "la participación activa de los padres en todos los aspectos de desarrollo social, emocional y académico de sus hijos" (p. 34); abarca las expectativas de los padres sobre el futuro académico de sus hijos, el control e involucramiento en la realización de las tareas o la frecuencia con la que los padres se presentan en la escuela.

Un componente multicitado en torno a la implicación familiar son las expectativas familiares sobre el éxito escolar, que han sido estudiadas de manera amplia porque relacionan la influencia de las creencias y los valores familiares con los resultados académicos, y se constituyen como una importante forma de socializar el valor de la educación al interior de los hogares; además, se ha identificado que las expectativas tienen consecuencias sobre la autopercepción de las propias capacidades como estudiantes, así como con una mayor motivación para desempeñarse en la escuela. En este sentido, Mac Iver et al. (2015) sugieren que las familias cumplen un rol significativo en apoyar el desarrollo de los estudiantes, modelar comportamientos de aprendizaje y reforzar la importancia del trabajo escolar y el éxito en la escuela. Estas tareas adquieren un papel significativo durante la adolescencia y la juventud.

Con esta distinción en mente, es posible señalar que el concepto de involucramiento familiar en educación efectivamente considera actividades variadas que van desde la supervisión de conducta hasta la gestión escolar. Dado que es un constructo muy amplio para su estudio, como advierten Loya, Arzola y Armendáriz (2013), convendría distinguirlos en función de dos tipos de procesos:

-Procesos de aprendizaje-desarrollo de los estudiantes. En esta categoría se pueden incluir el logro académico, la permanencia escolar, el desarrollo de habilidades socioemocionales, y otras que contribuyan al aumento de las capacidades cognitivas, sociales, emocionales y morales de los educandos.

-Procesos de gestión escolar. Comprende la participación en organización de actividades extraescolares de los estudiantes, o bien, en asociaciones de padres de familia, cuya finalidad sea recabar recursos económicos para hacer mejoras a la infraestructura de la escuela o equiparla; de esta manera, se tiene mayor claridad sobre la intención y las finalidades de las interacciones familia-escuela.

Hasta aquí, identificamos la etapa evolutiva de los educandos como una condición para definir el concepto de implicación familiar, así como el tipo de actividades que resultan en beneficio de los procesos de aprendizaje de los estudiantes. Dicho de otro modo, la implicación familiar en educación básica se puede entender como una supervisión cercana de las actividades escolares, que privilegia actividades de crianza, como la protección y provisión de un ambiente educativo tanto en el hogar como en la escuela, mientras que la implicación familiar durante la educación media se ve como una guía u orientación, una plataforma de apoyo brindada por la familia, que cobra mayor significado cuando se expresan altas expectativas en torno a la escolarización, con lo que se da lugar al desarrollo de la autonomía de los jóvenes, quienes

Infante / Padilla. Implicación familiar en el bachillerato: una estrategia para favorecer la permanencia escolar Sinéctica 54 www.sinectica.iteso.mx 
son cada vez más capaces de tomar y asumir las consecuencias de sus decisiones académicas y personales.

\section{LA IMPLiCACión FAMILIAR COMO ESTRATEgIA PARA LA PERMANENCIA ESCOLAR EN EL BACHILLERATO}

Como mencionamos en la introducción, el tema de la permanencia escolar en educación media superior supone un gran reto. Las tasas de deserción indican que, en México, aproximadamente setecientos mil jóvenes dejan la escuela cada año (14.4\%), la más alta cifra de deserción del Sistema Educativo Nacional (INEE, 2017). Esta realidad es común en otros países de la región latina, pues, según Bassi, Busso y Muñoz (2013), a excepción de Chile, los países de América Latina muestran bajo índice de graduación en el bachillerato: un $60 \%$ se gradúa y solo $46 \%$ termina sus estudios en el tiempo establecido.

Estos datos y el interés de las políticas educativas por impulsar la obligatoriedad de la escuela media revelan que la permanencia escolar es un tema que ocupa las agendas políticas de los países de la región. En este apartado mostramos la relación entre permanencia escolar e implicación familiar con base en algunos hallazgos revelados por las investigaciones identificadas en la literatura. Asimismo, damos cuenta de las políticas educativas, que, en el caso mexicano, han destacado la participación familiar como un medio para promover la permanencia escolar en el bachillerato.

El fenómeno de la deserción se ha catalogado como un proceso "multifactorial, complejo, dinámico y acumulativo" (Weiss, 2014, p. 32), que comprende aspectos sociales, educativos, familiares e individuales. Entre las causas asociadas a la deserción, en primer lugar está la económica o social; en segundo, la académica o educativa; y en tercero, la familiar o personal (Weiss, 2014). Los especialistas apuntan la importancia de fomentar la participación de las familias en la formación educativa de los jóvenes, ya que es un elemento que, además de influir en su rendimiento académico, aumenta la posibilidad de que continúen estudiando y evita que abandonen la escuela (Weiss, 2015).

Por su parte, la Encuesta Nacional de Deserción en Media Superior (ENDEMS) también señala que las prácticas y creencias familiares en torno a la educación pueden ser un factor asociado a la deserción y, por ello, reconocen que dentro de los mecanismos de intervención se debe incorporar a las familias, la comunidad estudiantil y al resto de la sociedad; sostiene que el establecimiento de una comunicación cercana entre la escuela y la familia disminuye la posibilidad de deserción (SEP, SEMS y COPEEMS, 2012).

La evidencia empírica mostrada por Terry (2008) confirma la influencia familiar en la decisión de jóvenes al abandonar la escuela. Desde las voces de jóvenes desertores, se sabe que hubo ausencia de apoyo familiar en la vida escolar y actitudes indiferentes de sus familias hacia la escuela propiciados por ambientes familiares de abuso de drogas y violencia, manifestados como alicientes para dejar la escuela; de igual forma, se revela una alta probabilidad de que los jóvenes abandonen la escuela si algún miembro de sus familias también fue desertor. Esto sugiere que las creencias familiares en torno al valor de la educación se pueden manifestar en prácticas que favorezcan el abandono o la permanencia escolar. 
En México, desde 2013, se ha implementado el movimiento "Yo no abandono" como estrategia nacional para favorecer la permanencia de los estudiantes de bachillerato, cuyo eje es la reunión de los esfuerzos de docentes, estudiantes y padres de familia en la prevención del abandono escolar y el fomento de la permanencia escolar (SEP, 2015). Esta estrategia ofrece una caja de doce herramientas que aborda temas relacionados con la prevención de los riesgos del abandono escolar, la introducción a estudiantes de nuevo ingreso, el acompañamiento de las decisiones de los jóvenes, el acercamiento con los padres de familia, el mejoramiento de la impartición de tutorías y la puesta en marcha de un proceso de planeación participativa, con el objetivo de apoyar el trabajo de todos los actores involucrados.

En lo anterior, sobresale la temática de la relación entre escuelas y padres de familia como una estrategia contra el abandono. En ella se ofrece una guía para que directivos y profesores incentiven el diálogo con las familias, en especial de aquellos estudiantes en riesgo de abandono. Las orientaciones giran en torno al reconocimiento de la necesidad de comunicación permanente entre los bachilleratos y las familias; esto se traduce en actividades que comprenden la disposición de directivos y docentes para atender los comentarios y conversar con las familias, individual o grupalmente, cuando estas así lo requieran, además del envío de citatorios por escrito para organizar reuniones, así como la intervención de los padres en actividades académicas, deportivas o culturales (SEMS, 2014).

Como mencionamos, según Weiss (2015), las variables familiares asociadas al contexto socioeconómico están dentro de las tres primeras razones por las que los jóvenes abandonan la educación media superior, de ahí que se propone mirar a las familias como un actor que contribuya a enfrentar esta problemática. Mejorar la relación entre estas y los bachilleratos se vislumbra como un camino que contribuye a enfrentar las dificultades de deserción que se advierten en este nivel educativo.

En consonancia con este planteamiento, el Instituto Nacional para la Evaluación de la Educación (INEE, 2017), sumado a la iniciativa de diferentes programas para favorecer la permanencia escolar en EMS, publicó las Directrices para mejorar la permanencia escolar en EMS; igual que el movimiento "Yo no abandono", la propuesta del INNE también explica la necesidad de mejorar la comunicación entre escuela y familia. Esto responde a la exigencia de intervenir con acciones concretas que aparten a los jóvenes de situaciones que los lleven a dejar la escuela; para ello, la familia se reconoce como aliada por su interés en que el joven continúe estudiando, al ser parte de su núcleo cercano y por la influencia que puede tener en sus decisiones académicas.

Las familias son una fuente de apoyo importante en el logro de la permanencia en la EMS, pues el interés por asistir a la escuela puede estimularse en los estudiantes, en tanto observen que terminar el bachillerato es un asunto relevante para sus familias. Para la sociedad mexicana, la educación media posee un alto valor (Weiss, 2012). En razón de ello, conviene involucrar a las familias en la cultura escolar del bachillerato durante los primeros semestres para establecer una alianza que permita crear sentido de pertenencia al bachillerato entre los jóvenes y sus familias (ENDEMS, 2012).

Infante / Padilla. Implicación familiar en el bachillerato: una estrategia para favorecer la permanencia escolar Sinéctica 54 www.sinectica.iteso. $m x$ 


\section{Prácticas FAMILIARES QUE CONTRIBUYEN AL LOGRO ACADÉMICO DE LOS BACHILLERES}

Diversos análisis confirman que la implicación familiar tiene un impacto positivo en el logro académico de los estudiantes (Castro et al., 2015; Crosnoe, 2001; Fan \& Chen, 2001; Fernández-Alonso et al., 2017; Hill \& Tyson, 2009; Jeynes, 2007, 2011; Pomerantz, Moorman \& Litwack, 2007; Redding, 2006; Wilder, 2014). Debemos señalar que la mayoría de los trabajos revisados se realizaron en países anglosajones y en España.

Lo anterior, además de un mayor interés por el estudio de este fenómeno, deja ver que el involucramiento familiar en la educación escolarizada tiende a darse en dos condiciones importantes: la primera referida al interés de las escuelas en trabajar de manera conjunta con las familias, y la segunda, al valor de la escolarización por parte de las familias. Estas condiciones del contexto pueden propiciar interacciones armónicas entre familias y escuelas, lo que ha arrojado resultados satisfactorios para las comunidades escolares. Estas podrían ser las condiciones ideales para que se dé la relación familia-escuela; sin embargo, conviene tener en cuenta que no siempre se presentan con la intensidad, frecuencia u oportunidad requeridas, y que ello dará lugar a distintos matices de relación entre familias y escuelas, sobre todo en un país como México, en el que la desigualdad social abre grandes brechas sociales.

Estos trabajos estudian la implicación familiar mediante diferentes acercamientos, así como las dificultades al delimitar prácticas generalizadas que influyen en el logro académico. No obstante, coinciden en que las prácticas de involucramiento familiar en la etapa adolescente se modifican sustancialmente ante la creciente necesidad evolutiva del estudiante por reafirmar su independencia, las creencias de los padres sobre su rol educativo $y$, a su vez, las condiciones que promueve el bachillerato para vincularlos con las familias. En contraste, los estudiantes de educación básica se ven más influenciados por los valores familiares durante los primeros años de vida, mientras que los bachilleres están más convencidos de sus propias fortalezas y debilidades académicas. En efecto, la implicación familiar en el bachillerato sigue siendo importante para el logro académico, aunque las prácticas cambian para dar lugar al desarrollo de la autonomía en el adolescente.

En relación con los procesos de aprendizaje, el logro académico funciona como un indicador que refleja, a menudo, los niveles de aprendizaje de los estudiantes. Aunque es un concepto amplio que abarca el dominio de diferentes asignaturas, su definición depende de los indicadores con que se mide, los cuales pueden estar centrados en el proceso de aprendizaje, en el currículo o ser acumulativos, y se pueden traducir en aprendizajes declarados, calificaciones, puntajes de pruebas estandarizadas, permanencia escolar y culminación de grados educativos; en suma, estos criterios tienen en común que se refieren al logro académico, como el esfuerzo y la capacidad intelectual del estudiante. En términos de la implicación familiar, el logro académico significa que el educando está desarrollando su máximo potencial, apoyado en las condiciones familiares, las cuales provean un ambiente adecuado para ello. 
El logro académico ha sido foco de interés de muchos campos del conocimiento, pues se ha vinculado fuertemente al desarrollo socioeconómico de las comunidades; prueba de ello han sido las evaluaciones internacionales, como PISA (Steinmayr \& Spinath, 2007).

La hipótesis de que la implicación familiar tiene efectos positivos para el logro académico ha sustentado investigaciones que encuentran correlaciones entre estas dos variables, desde la sostenida por los estudios de Coleman, en los que "el capital social está asociado a una menor deserción escolar y, en general, a un mayor logro educativo" (Ramírez y Hernández, 2012, p. 1). Por la dificultad de acotar el término de implicación familiar y las diferentes mediciones que se hacen del logro académico, el análisis de Wilder (2014) sugiere que las evaluaciones del logro académico en relación con la implicación familiar se sitúan en dos categorías: estandarizadas y no estandarizadas. Las medidas estandarizadas se entienden como los resultados en pruebas de rendimiento; las no estandarizadas incluyen la valoración de los profesores sobre el rendimiento y comportamiento de estudiantes, tasa de tareas completadas, hábitos y actitudes hacia el estudio.

Uno de los cambios más significativos en las prácticas de implicación familiar en el bachillerato es el referente a las tareas escolares. La investigación de FernándezAlonso et al. (2017) muestra correlaciones negativas entre una supervisión cercana al elaborar tareas escolares y el logro académico. Esto apunta a que, en la medida en que el estudiante va adquiriendo autonomía para realizar las tareas escolares, la presencia de la familia disminuye, pues el trabajo y la responsabilidad de un estudiante sobre sus actividades escolares son la clave del logro académico.

También sobre las prácticas de involucramiento familiar en el bachillerato, el metaanálisis de Hill y Tyson (2009) arroja datos concluyentes sobre las formas más exitosas de involucramiento en lo concerniente al logro académico y desarrollo cognitivo de los adolescentes. Las autoras presentan tres categorías que agrupan distintas prácticas de implicación familiar en el bachillerato: involucramiento en actividades escolares, que incluye la asistencia a los actos que organiza la escuela para involucrar a los padres; involucramiento cognitivo-intelectual, traducido en el apoyo que los padres brindan en la realización de tareas escolares; e involucramiento personal, que se refiere a la socialización acerca del valor de la educación. Estas categorías son analizadas en función del logro académico y apuntalan tres importantes hallazgos:

-El involucramiento familiar que crea una comprensión sobre los propósitos y el significado del rendimiento académico comunica expectativas educativas y proporciona estrategias que los estudiantes pueden usar efectivamente; se relaciona de manera positiva con el logro académico (socialización sobre el valor escolar).

- La respuesta familiar a las actividades promovidas por la escuela mantiene una relación positiva-moderada con el logro académico.

-El involucramiento familiar vinculado a la supervisión de la tarea no se relaciona con el logro académico de un modo consistente.

Los resultados del estudio identifican el papel de la autonomía en relación con la participación familiar en bachillerato; precisan que, en educación media, sí puede

Infante / Padilla. Implicación familiar en el bachillerato: una estrategia para favorecer la permanencia escolar Sinéctica 54 www.sinectica.iteso. $m x$ 
haber desarrollo de la autonomía, independencia y habilidades cognitivas a la par de un involucramiento familiar que privilegia la socialización del valor educativo. Al mismo tiempo, se reconoce que la supervisión cercana de las tareas escolares deja de ser una práctica que influya positivamente en el logro académico.

De igual forma, Crosnoe (2001) especifica que en esta etapa la participación familiar en las tareas escolares puede disminuir, pero la comunicación dentro de la familia, manifestada en la socialización sobre el valor de la educación y las actividades de aprendizaje, adquiere un papel trascendente que apoya las trayectorias escolares. El mismo autor expone que el involucramiento familiar en el bachillerato puede dar lugar a una paradoja: cuando las familias observan la mejora en los resultados académicos, específicamente en las calificaciones, pueden obviar la necesidad de continuar participando de manera cercana. En este caso, las prácticas de implicación familiar otorgan mayor libertad y capacidad de decisión al estudiante, situaciones que no se convierten, de modo necesario, en desentendimiento de los asuntos escolares.

La comunicación al interior de la familia sobre el valor de la educación cobra un papel relevante en este nivel educativo, ya que, a través de ella, se transmiten las expectativas en torno al grado educativo que se espera de los estudiantes. Se percibe relación entre el tipo de implicación familiar y el valor que la educación puede tener para una comunidad; si este es visto como una oportunidad de movilidad social y desarrollo, es posible que la familia emprenda acciones que permitan un tránsito adecuado en el nivel medio superior.

Retomamos el modelo de Epstein (2010), cuya propuesta sostiene seis modalidades de implicación familiar, con el propósito de integrar los planteamientos anteriores y visualizar las aportaciones para cada categoría. Desde la perspectiva sistémica de este modelo, el centro de la relación familia, escuela y comunidad es el estudiante; así, la finalidad de esta alianza, además de proporcionar un ambiente educativo que favorezca su aprendizaje, es conducir esfuerzos para motivarlo y guiarlo al desarrollo de su potencial. De esta manera, se llega a una postura completa sobre la implicación familiar en el bachillerato, que no solo se circunscribe al desempeño de los contenidos escolares, sino que incorpora el fomento de las capacidades del estudiante.

La primera modalidad planteada es la crianza, que se refiere al establecimiento de condiciones en el entorno del hogar que favorezcan un ambiente físico y emocional propicio para el estudio; se equipara con el concepto de currículo del hogar, propuesto por Redding (2006, p. 7), quien lo define como "las relaciones, las prácticas y los patrones de vida familiar", expresiones con las que la familia promueve la obtención de actitudes y hábitos positivos hacia el estudio.

La segunda modalidad es la comunicación, con la cual se pretende establecer relación entre familias y escuela, entendida como las interacciones que permitan dar seguimiento a la formación escolar del estudiante, y manifestada en el conocimiento que tienen las familias sobre las rutinas escolares, las calificaciones, preferencias por contenidos específicos, necesidades de apoyo económico o de materiales de aprendizaje, entre otros (Mac Iver et al., 2015). 
La tercera es el voluntariado, concebido como el fomento y la organización del apoyo brindado por las familias en el desarrollo de las actividades del aula y la escuela; Simon (2004) señala que el bachillerato es el encargado de involucrar a todas las familias mediante actividades individuales o grupales que respondan a las necesidades de sus estudiantes.

El aprendizaje en el hogar es la cuarta modalidad; tiene que ver con la provisión de medios para la realización de tareas escolares. Esta dimensión ha vinculado el apoyo familiar en la elaboración de tareas con el logro académico, aunque para la educación media las investigaciones de Benner, Boyle y Sadler (2019), Boonk, Gijselaers, Ritzen y Brand-Gruwel (2018), Crosnoe (2001), Fernández-Alonso et al. (2017), Gonida y Cortina (2014), Hill y Tyson (2009) y Núñez et al. (2015) hacen hincapié en que esta relación no se da en la supervisión de las tareas; más bien se traduce en el apoyo económico que las familias proveen para la adquisición de materiales necesarios para ejecutar proyectos escolares, o bien, con el acercamiento a actividades culturales y recreativas que apoyen los aprendizajes escolares.

La quinta modalidad es toma de decisiones, que incluye a las familias en las decisiones escolares, al desarrollar su liderazgo y participación en las asociaciones y en los consejos de participación social (CPS); sobre este aspecto se hace mención de la Ley General de Educación (SEP, 1993) que, en México, determina, en su capítulo siete, los derechos y las obligaciones de las familias en relación con su participación en la educación, y se decretan las condiciones para conformar y operar los CPS en educación; el cumplimiento de esta normativa se extiende al bachillerato como parte de la educación obligatoria.

Esta modalidad ha sido abordada en diversos trabajos (Canales, 2006; Gonzaga, 2017; Latapí, 2004; Sabariegos, 2009; Santizo, 2011), que coinciden en la naciente existencia de la participación social en educación y destacan la participación de las familias como una condición para consolidar sociedades democráticas. El estudio empírico de James y Guzmán (2015) muestra la poca participación de las familias en escuelas secundarias mexicanas, la cual puede interpretarse en dos sentidos: como desinterés, o como evidencia de la compleja situación sindical de los profesores de educación básica. Revelan que los profesores tienen poca disposición para atender las inquietudes de los padres y que hay distancia entre estos y los padres de familia debido a sus ocupaciones laborales. Los autores concluyen que el silencio se convierte en la forma de participación por excelencia, pues los espacios para la expresión de la familia en la escuela son limitados y sus solicitudes pocas veces resueltas por los profesores.

Finalmente, la colaboración con la comunidad es la sexta modalidad; esta considera los recursos y servicios que tanto la comunidad como la escuela pueden brindarse mutuamente; se ve reflejado en la realización de algún tipo de servicio social comunitario por parte de los estudiantes desde el que se involucre también a las familias; ejemplo de ello puede ser organizar visitas a los orfanatos y ayudar a los menores con sus tareas, o bien, hacer brigadas para recoger basura o reforestar, entre otras; asimismo, la comunidad puede solicitar servicios de la escuela, como la participación de la banda de guerra en un acto particular o la convocatoria de un concurso de grafiti artístico para mejorar el aspecto urbano.

Infante / Padilla. Implicación familiar en el bachillerato: una estrategia para favorecer la permanencia escolar Sinéctica 54 www.sinectica.iteso.mx 
Estas actividades se llevan a cabo en el contexto mexicano, aunque con matices distintos según el tipo de bachillerato, sostenimiento, contexto y ubicación (zonas rurales y urbanas); se reconoce que esta modalidad ha sido poco estudiada en el ámbito mexicano. No obstante, en otras latitudes sí se ha logrado unificar a la familia, escuela y comunidad a través de esta clase de actividades; muestra de ello son los trabajos reportados por Harvard Family Research Project (2007), los cuales demuestran que, a través de la organización de los estudiantes y sus familias, se establecen nexos con la comunidad para abordar los problemas escolares y de la comunidad misma; estos esfuerzos han probado mejorar la perspectiva de los estudiantes y han ampliado su razonamiento moral, desarrollo cognitivo y social, así como su sentido de justicia social.

El análisis de este modelo de implicación familiar establece los puntos de encuentro entre familia y escuela, que pone de manifiesto el desarrollo de los educandos como un asunto que va más allá de las aulas y del entorno familiar, y que abarca a la comunidad misma. Observamos que la modalidad de aprendizaje es la más estudiada y las conclusiones que han derivado de estos estudios permiten tener mayor claridad sobre las prácticas familiares que favorecen el aprendizaje escolar; en cambio, otras como el voluntariado y la colaboración con la comunidad pueden considerarse modalidades que aún deben estudiarse con mayor profundidad, en razón de que estos tipos de implicación tienden a desarrollarse más en unos entornos que en otros; esto se debe a diferencias en la cultura participativa, por las que se valora en mayor o menor medida la colaboración como vehículo de desarrollo social.

\section{ESTRATEGIAS PARA ESTABLECER UN VÍNCULO EFECTIVO CON LAS FAMILIAS}

Como señalamos en el primer apartado, la relación familia-escuela se determina en dos direcciones: a través de las acciones que emprende la escuela y las que se llevan al interior de la familia; ambas refieren la creación de sinergia para potencializar las capacidades de los estudiantes. Una vez que hemos abordado algunas prácticas familiares que inciden en el logro académico, en este apartado condensamos algunas recomendaciones para bachilleratos, recuperadas de intervenciones que fomentan la promoción de la implicación familiar, cuyos resultados han mostrado ser exitosos. Las sugerencias se exponen en la tabla que incluimos y se agrupan tomando en cuenta las seis modalidades de implicación familiar del modelo Epstein (2010); se plantean apelando a que no son las únicas estrategias para mejorar la relación entre bachilleratos y familias, sino que cada plantel educativo podrá crear las propias de acuerdo con su contexto y necesidades.

La sugerencia para la primera modalidad tiene que ver con el acompañamiento que los bachilleratos pueden ofrecer a las familias en relación con la parentalidad, de tal forma que con estas intervenciones, bachillerato y familia enfrenten, de manera conjunta, las problemáticas características de los estudiantes. Las actividades de la segunda y tercera modalidad se plantean el establecer contacto con las familias y que, a su vez, les reconozcan en las actividades formativas de los educandos, así como la incorporación del uso tecnología e internet, a través de plataformas educativas y aplicaciones, lo que puede facilitar la comunicación en tiempo real. 
Las intervenciones de la modalidad aprendizaje en el hogar buscan involucrar a las familias en la creación de ambientes educativos que se extiendan más allá de las aulas, en los cuales se complemente el desarrollo de las competencias planteadas por el modelo educativo. La quinta modalidad invita a los bachilleratos a despertar el interés genuino de las familias por la participación social en educación, entendiendo que esta no solo es relativa a la gestión de recursos, sino en general a la mejora de los centros educativos, entre los que se puede incluir el clima escolar.

En la sexta modalidad se propone integrar a las familias en actividades sociales que posiblemente los estudiantes ya estén llevando a cabo; se trata de vincular a las familias empleando sus contactos e ideas para, así, conectar de mejor manera con las necesidades más próximas al contexto escolar.

Reconocemos la capacidad y agencia de los bachilleratos en el fomento de actividades que impliquen a las familias a fin de impactar en el desarrollo intelectual, físico, emocional y social de los estudiantes. La elaboración de estas estrategias puede contribuir a concienciar a las escuelas sobre su papel en la generación y el mantenimiento de lazos con las familias (Egido, 2015; Simon, 2004).

De manera similar, el Ministerio de Educación, Cultura y Deporte (2014) propone que, para que las tareas de involucramiento familiar tengan mayor alcance, es preciso integrar, durante la formación o actualización del docente, temas sobre los beneficios de la implicación familiar para que, poco a poco, se incorporen a las prácticas de planeación y didáctica docente. Lo anterior puede crear un clima de cooperación que evidencie el compromiso de la escuela con el desarrollo de los estudiantes y que, al mismo tiempo, estimule en las familias un sentido de pertenencia hacia la escuela; ambos son catalizadores de la implicación familiar. En la misma dirección, hacemos un llamado a fortalecer los programas de tutorías, pues en EMS son los tutores quienes brindan constante cercanía con las problemáticas y los progresos de sus estudiantes; su acompañamiento puede ser visto como el puente de contacto con las familias. 
Tabla. Recomendaciones para la mejora de la implicación familiar en bachilleratos

\begin{tabular}{|c|c|}
\hline Modalidad & Recomendaciones \\
\hline Crianza & $\begin{array}{l}\text { Implementación de charlas y talleres en los que, con la colaboración de especialistas, se brinde } \\
\text { asesoría a las familias sobre temas socioemocionales, de salud reproductiva, nutrición, preven- } \\
\text { ción de adicciones, entre otros }\end{array}$ \\
\hline Comunicación & $\begin{array}{l}\text { Realizar al menos dos reuniones con familiares durante el ciclo escolar. La reunión inicial con } \\
\text { la finalidad de establecer contacto, presentar al personal, brindar información clara sobre las } \\
\text { materias, la forma de calificación, actividades extracurriculares y reglamento de la institución; } \\
\text { de igual manera, a partir de esa reunión, es oportuno crear un directorio con los datos de } \\
\text { contacto de cada familia para que, en caso de ser necesario, se le pueda convocar de modo } \\
\text { particular }\end{array}$ \\
\hline \multirow{3}{*}{ Voluntariado } & $\begin{array}{l}\text { Extender la invitación para que las familias participen en reuniones de docentes y directivos; } \\
\text { con ello, sensibilizarlas sobre las principales problemáticas que enfrentan e idear juntos for- } \\
\text { mas de resolverlas }\end{array}$ \\
\hline & $\begin{array}{l}\text { Ofrecer un espacio para que las familias puedan reunirse de manera voluntaria en la escuela } \\
\text { que les permita planificar e implementar acciones para la mejora del plantel }\end{array}$ \\
\hline & $\begin{array}{l}\text { Asignar una comisión de familias que se encarguen de la organización de actividades deporti- } \\
\text { vas y culturales, cuya finalidad es, a través de actividades recreativas, promover la integración } \\
\text { de la comunidad educativa }\end{array}$ \\
\hline \multirow{3}{*}{ Aprendizaje en casa } & $\begin{array}{l}\text { Brindar información a las familias en un lenguaje sencillo sobre las habilidades que los estu- } \\
\text { diantes deberán lograr en cada grado y cómo estas pueden complementarlas }\end{array}$ \\
\hline & $\begin{array}{l}\text { Involucrar a las familias en el proceso de decisión de carrera profesional o de actividad laboral, } \\
\text { que el estudiante vive durante el bachillerato, por medio de actividades charlas o sesiones de } \\
\text { orientación conjuntas }\end{array}$ \\
\hline & $\begin{array}{l}\text { Estimular la participación en familia a actos culturales que se desarrollan en la comunidad, } \\
\text { como asistencia a conciertos, exposiciones, museos u otros }\end{array}$ \\
\hline Toma de decisiones & $\begin{array}{l}\text { Promover la conformación de una asociación de familias representantes, destinada a conse- } \\
\text { guir recursos para la mejora de la escuela }\end{array}$ \\
\hline $\begin{array}{l}\text { Colaboración con la } \\
\text { comunidad }\end{array}$ & $\begin{array}{l}\text { Involucrar a las familias en la conformación de grupos estudiantiles que presten algún tipo de } \\
\text { servicio social comunitario }\end{array}$ \\
\hline
\end{tabular}

Fuente: Elaboración propia con base en las propuestas de Egido (2015), Epstein (2010) y Ministerio de Educación, Cultura y Deporte (2014).

En los bachilleratos, especialmente en los mexicanos, converge una diversidad de familias, cuyas características de estructura y capital cultural son muy amplias. Egido (2015) sugiere que, para el éxito de los programas de implicación familiar, es imprescindible considerar a padres y profesores como iguales, y rescatar el valor de sus aportaciones al desarrollo del estudiante; con esto en mente, elaborar un plan de trabajo cooperativo basado en el diálogo y la mutua confianza. Sobre esta base, se deberá buscar la colaboración de todas las familias, y poner especial atención en las que poco se involucran, considerando sus particularidades y mostrando una actitud de flexibilidad y apertura. También, reconocemos que la implicación familiar es un asunto de calidad más que de cantidad, por lo que la planificación de las actividades es necesaria, siempre tomando en cuenta su finalidad y durabilidad a través del tiempo.

\section{Discusión}

Las ideas aquí mostradas refieren que la vinculación de las familias con la educación media es importante, pues proporciona a los estudiantes un entorno formativo completo que favorece su permanencia en el bachillerato. Desarrollamos una visión amplia del concepto de implicación familiar cuya finalidad no se reduce al ámbito 
académico, reconocido como logro educativo; asimismo, destacamos que tanto familias como escuelas están llamadas a entablar comunicación y establecer relaciones efectivas en aras del desarrollo del bachiller.

Nuestro estudio recupera el modelo de Epstein (2010) para analizar los trabajos que abordan la implicación familiar en educación media. En relación con las modalidades de voluntariado, toma de decisiones y colaboración con la comunidad, observamos que pudieran no estar representadas en su totalidad en las escuelas mexicanas, pues hacen alusión a una cultura altamente participativa, que no se identifica en esta región de modo necesario. Sin embargo, lograr una vinculación entre la familia, la escuela y la comunidad, mediante el interés por los asuntos educativos, representa el máximo grado de colaboración en beneficio de los estudiantes, lo que, por supuesto, se convierte en el objeto de todas las prácticas de involucramiento familiar.

En este sentido, advertimos que la clase socioeconómica de las familias puede limitar o favorecer el desarrollo de actitudes socioafectivas y cognitivas de los educandos. Las investigaciones que aquí presentamos se desarrollaron, en su mayoría, en contextos distintos al mexicano, por lo que valdría la pena iniciar nuevos estudios sobre las prácticas de involucramiento familiar situados en la realidad del país, como el que proponen James y Guzmán (2015).

Las prácticas de implicación familiar mostradas en la tabla representan estrategias con las que los bachilleratos podrían mejorar la interacción con las familias. Aunque es posible que algunas de ellas ya se lleven a cabo, pueden llegar a estar desgastadas, como ejemplo se citan las reuniones para firmar boleta de calificaciones o la existencia de asociaciones de padres de familia, con una limitada participación y reconocimiento por parte de la comunidad educativa.

La realización de actividades para involucrar a las familias en la educación de los bachilleres es algo que no debe hacerse por inercia; requiere una adecuada planificación y ejecución, teniendo siempre como propósito integrar ambientes de aprendizaje para los jóvenes; de lo contrario, resulta en prácticas anquilosadas con limitada efectividad; así, buscamos que las escuelas generen un sentido de pertenencia que se extienda a estudiantes y sus familias. Con ello, resaltamos el carácter preventivo de la implicación familiar, que favorece la permanencia de los estudiantes en el bachillerato; esto evita la idea de concebirla como una acción remedial.

Respecto a la modalidad de aprendizaje en el hogar, evidentemente se hace presente un cambio en la forma en que la familia se involucra en la realización de la tarea, pues, a diferencia de la educación básica, en el bachillerato se prescinde de una supervisión cercana, y se da paso al desarrollo de la autonomía del joven. En este tipo educativo, resulta favorable para el estudiante que la familia le procure los insumos necesarios para ejecutar proyectos escolares, por ejemplo, la provisión de un espacio adecuado, un dispositivo electrónico con acceso a internet, libros, etcétera; estas acciones comunican al estudiante el interés de la familia por su aprendizaje.

Por otro lado, es importante emprender investigaciones que den cuenta de la implicación familiar en el bachillerato en lo referente al desarrollo de la autonomía del joven, ya que esto la distingue de la implicación familiar en la educación básica. Los planteamientos antes revisados sugieren que sí es posible una sana relación entre familias y bachilleratos, en la que se privilegie el acompañamiento familiar y en la 
que las familias expliquen el valor educativo y ayuden al bachiller a definir su rol como estudiante. Especialmente en la etapa en que se da la transición a la educación superior, la familia contribuye a la adquisición de la autonomía del joven (PadillaGonzález, Figueroa y Rodríguez-Figueroa, 2017).

La revisión bibliográfica confirma la necesidad de ampliar el número de estudios que analicen la temática de la implicación familiar en el bachillerato, pues aún se desconocen cuáles son las condiciones que las escuelas ofrecen para incluir a las familias en la formación de los estudiantes, qué acciones concretas llevan a cabo las familias para promover que los jóvenes continúen sus estudios y qué prácticas favorecen sus aprendizajes; todo ello en un marco contextualizado en el que se reconozca la complejidad que caracteriza a los subsistemas y modalidades del bachillerato mexicano.

Abordar esta temática también supone otorgar especial atención a los aspectos culturales, los cuales definen el interés de las instituciones sociales por la participación en la educación de sus niños y jóvenes; así, es de esperar que los niveles de implicación familiar en el bachillerato sean distintos de acuerdo con la comunidad a la que pertenezca la escuela, las zonas urbanas y rurales, la escolaridad de las familias y los imaginarios que se compartan respecto al valor de la educación.

\section{REFERENCIAS BIBLIOGRÁFICAS}

Bassi, M., Busso, M. \& Muñoz, J. S. (2013). Is the glass half empty or half full? School enrollment, graduation, and droput rates in Latin America. Political Science, Art. doi:10.2139/ssrn.2367706

Benner, A. D., Boyle, A. E. \& Sadler, S. (2016). Parental Involvement and adolescents' educational success: The roles of prior achievement and socioeconomic status. Journal of Youth and Adolescence, vol. 45, núm. 6. https://doi. org/10.1007/s10964-016-0431-4

Boonk, L., Gijselaers, H. J. M., Ritzen, H. \& Brand-Gruwel, S. (2018). A review of the relationship between parental involvement indicators and academic achievement. Educational Research Review, vol. 24. https://doi.org/10.1016/j.edurev.2018.02.001

Bustamante Diez, Y. (2014). La educación media superior en México. Innovación Educativa, vol. 14, núm. 64, pp. 11-22.

Canales, S. A. (2006). La participación social en educación: un dilema por resolver. Perfiles Educativos, vol. 28, núm. 113, pp. 64-80.

Castro, M., Expósito-Casas, E., López-Martín, E., Lizasoain, L., Navarro-Asencio, E. \& Gaviria, J. L. (2015). Parental involvement on student academic achievement: A meta-analysis. Educational Research Review, vol. 14, pp. 33-46. https://doi.org/10.1016/j.edurev.2015.01.002

Comellas, M. J., Missio, M., Sánchez, L., Garcia, B., Bodner, N., Casals, I. \& Lojo, M. (2013). Familia, escuela y comunidad: un encuentro necesario. Barcelona: Octaedro.

Crosnoe, R. (2001). Academic orientation and parental involvement in education during high school. Sociology of Education, vol. 74, núm. 3, pp. 210-230. https://doi.org/10.2307/2673275 
Desforges, C. \& Abouchaar, A. (2003). The impact of parental involvement, parental support and family education on pupil achievements and adjustment: A literature review with. Research Report 433. Reino Unido. https://doi. org/10.1016/j.ctrv.2004.06.001

Egido, G. I. (2015). Participación educativa. Participación Educativa. Revista Del Consenjo Escolar Del Estado, vol. 4, núm. 7, pp. 11-17.

Epstein, B. J. L. (2010). School family community parterships. Phi Delta Kappan, vol. 76, núm. 9, pp. 701-712.

Fan, X. \& Chen, M. (2001). Parental involvement and studnet's academic achievement: a meta-analysis. Educational Psychology Review, vol. 13, núm. 1, pp. 1-22. https://doi.org/10.1023/A:1009048817385

Fernández-Alonso, R., Álvarez-Díaz, M., Woitschach, P., Suárez-Álvarez, J. \& Cuesta, M. (2017). Parental involvement and academic performance: Less control and more communication. Psicothema, vol. 29, núm. 4, pp. 453-461. https:// doi.org/10.7334/psicothema2017.181

Flecha, R. (2015). Successful educational actions for inclusion and social cohesion in Europe. Barcelona: Springer. https://doi.org/10.1007/978-3-319-11176-6

Glasgow, N. A. \& Whitney, P. J. (2009). What sucessful schools do to involve families. Londres: Sage.

Gonida, E. N. \& Cortina, K. S. (2014). Parental involvement in homework: Relations with parent and student achievement-related motivational beliefs and achievement. British Journal of Educational Psychology, vol. 84, núm. 3. https://doi.org/10.1111/bjep.12039

Harvard Family Research Project. (2007). Family involvement makes a difference. Evidence that family promotes school success for every child of every age. Family involvement in middle and high school students'education. Recuperado de www.hfrp.org.

Hernández, P. M., Gomariz, V. M.A., Parra, M. J. y García, S. M. P. (2016). Familia, inmigración y comunicación con el centro escolar: un estudio comparativo. Educacion XX1, vol. 19, núm. 2 (Educación XXI), pp. 127-151. https://doi. org/10.5944/educXX1.14229

Hill, N. E. \& Tyson, D. F. (2009). Parental involvement in middle school: A Meta-Analytic assessment of the strategies that promote achievement. Developmental Psychology, vol. 45, núm. 3, pp. 740-763. https://doi.org/10.1037/ a0015362

Huerta, V. E. (2009). La relación escuela-padres en las secundarias mexicanas. Colección Cuadernos de Investigación. Recuperado de www.inee.edu.mx

INEE (2017). Panorama educativo de México 2016. Indicadores del sistema educativo nacional 2016. Educación básica y media superior. Ciudad de México.

James, C. M. y Guzmán, F. E. (2015). La participación de madres y padres de familia en la escuela: un divorcio de mutuo consentimiento. Sinéctica, Revista Electrónica de Educación, vol. 46, pp. 1-23. Recuperado de http://www.sinectica.iteso.mx/articulo/?id=46_la_participacion_de_madres_y_padres_de_familia_en_la_escuela_un_divorcio_de_mutuo_consentimiento

Jeynes, William H. (2011). Parental involvement and academic succes. Nueva York: Routlege. 
Jeynes, William H. (2007). The relationship between parental involvement and urban secondary school student academic achievement: A meta-analysis. Urban Education, vol. 42, pp. 82-110.

Latapí, S. P. (2004). La participación de la sociedad en la definición de políticas públicas de educación: el caso del Observatorio Ciudadano de la Educación. Perfiles Educativos, vol. XXVII, pp. 7-39.

Loya, O. C. G., Arzola, F. D. M. y Armendáriz, P. H. M. (2013). La participación de los padres de familia en el ámbito de la Rieb. Revista de Investigación Educativa de La REDIECH, vol. 4, núm. 7, pp. 33-42.

Mac Iver, M. A., Epstein, J. L., Sheldon, S. B. \& Fonseca, E. (2015). Engaging families to support students' transition to high school: Evidence from the field. The High School Journal, vol. 99, núm. 1, pp. 27-45. https://doi.org/10.1353/ hsj.2015.0016

Martínez Espinosa, M. A. (2012). Presentación. La eduación media hoy en día: perspectiva histórica. En La educación media superior en México (pp. 9-18). Ciudad de México: FCE/SEP/SEMS.

Ministerio de Educación, Cultura y Deporte (2014). La participación de las familias en la educación escolar. Madrid.

Núñez, J. C., Suárez, N., Rosário, P., Vallejo, G., Valle, A. \& Epstein, J. L. (2015). Relationships between perceived parental involvement in homework, student homework behaviors, and academic achievement: differences among elementary, junior high, and high school students. Metacognition and Learning, vol. 10, núm. 3. https://doi.org/10.1007/s11409-015-9135-5

O'Shea, S. (2016). Avoiding the manufacture of 'sameness': First-in-family students, cultural capital and the higher education environment. Higher Education, vol. 72, pp. 59-78. https://doi.org/10.1007/s10734-015-9938-y

Padilla-González, L. E., Figueroa, Ruvalcaba, A. E. y Rodríguez-Figueroa, H. M. (2017). La incorporación a la universidad de los estudiantes en Aguascalientes. La perspectiva del orientador educativo. Sinéctica, Revista Electrónica de Educación, núm. 48, pp. Recuperado de https://sinectica.iteso.mx/index. php/SINECTICA/article/view/641/716

Pomerantz, E. M., Moorman, E. A. \& Litwack, S. D. (2007). The how, whom, and why of parents' involvement in children's academic lives: More is not always better. Review of Educational Research, vol. 77, núm. 3, pp. 373-410. https:// doi.org/10.3102/003465430305567

Ramírez, J. y Hernández, E. (2012). ¿Tenía razon Coleman? Acerca de la relación entre capital social y logro educativo. Sinéctica, Revista Electrónica de Educación, núm. 39.

Redding, S. (2000). Familias y escuelas. Ginebra: Academia Internacional de Educación y Oficina Internacional de Educación. Recuperado de http://www. ibe.unesco.org

Reparáz, R. y Naval, C. (2014). Bases conceptuales de la participación de las familias. En La participación de las familias en la educación escolar (pp. 21-34). Madrid: Ministerio de Educación, Cultura y Deporte.

Sabariegos, D. J. (2009). Participación social: planteamiento y evaluación de un modelo estructural a partir de variables observadas. Social Participation: Proposal and Evaluation of a Structural Model from Observed Variables, vol. 3, pp. 31-41. Recuperado de http://hdl.handle.net/10045/11440 
Sanders, M. G. \& Epstein, J. L. (2005). School-family-community partnerships and educational change: International perspectives. En Extending educational change: International Handbook of Educational Change (pp. 202-222). Holanda. https://doi.org/10.1007/1-4020-4453-4_10

Santizo, R. C. (2011). Gobernanza y participación en escuela pública. Revista Mexicana de Investigacion Educativa, vol. 16, núm. 50, julio-septiembre, pp. 751-773.

SEMS (2014). Yo no abandono. Manual para incentivar el diálogo con los padres de familia en planteles de educación media superior. México, DF: Progreso.

SEP (2015). Análisis del movimiento contra el abandono escolar en la educación media superior. Recuperado de http://www.sems.gob.mx/work/models/ sems/Resource/11390/8/images/reporte_abandono.pdf

SEP (1993). Ley General de Educación, Pub. L. núm. acuerdo 716.

SEP, SEMS y COPEEMS (2012). Reporte de la Encuesta Nacional de Deserción en la Educación Media Superior. Recuperado de http://www.sems.gob.mx/ work/models/sems/Resource/10787/1/images/Anexo_6Reporte_de_la_ ENDEMS.pdf

Simon, B. S. (2004). High school outreach and family involvement. Social Psychology of Education, vol. 7, núm. 2, pp. 185-209. https://doi.org/10.1023/ B:SPOE.0000018559.47658.67

Steinmayr, R. \& Spinath, B. (2007). Predicting school achievement from motivation and personality. Zeitschrift Für Pädagogische Psychologie [German Journal of Educational Psychology], vol. 21, pp. 207-216.

Terry, M. (2008). The effects that family members and peers have on students' decisions to drop out of school. Educational Research Quarterly, vol. 31, núm. 3, pp. 25-38.

Weiss, E. (2015). El abandono escolar en la educación media superior: dimensiones, causas y políticas para abatirlo. En Desafíos de la educación media superior (pp. 81-160). Ciudad de México: Instituto Belisario Dominguez.

Weiss, E. (2014). El abandono escolar en la educación media superior. México: Dirección General de Investigación Estratégica Instituto Belisario Domínguez Senado de la República.

Weiss, E. (coord.) (2012). Jóvenes y bachillerato. Biblioteca de la Educación Superior. Ciudad de México: ANUIES.

Wilder, S. (2014). Effects of parental involvement on academic achievement: A meta-synthesis. Educational Review, vol. 66, núm. 3, pp. 377-397. https:// doi.org/10.1080/00131911.2013.780009 
Infante / Padilla. Implicación familiar en el bachillerato: una estrategia para favorecer la permanencia escolar Sinéctica 54 www.sinectica.iteso.mx 